\section{AL-AZHAR Dental Journal}

F o r

\title{
Evaluation of Root Length Accompanying Platelet Rich Plasma Injection as a Technique for Orthodontic Tooth Movement Acceleration. (A Comparative Study)
}

\author{
Heba A. Seddik ${ }^{1 *}$, Samir A. Ibrahim ${ }^{2}$, and Mai S. Attia ${ }^{3}$
}

\begin{tabular}{|l|}
\hline Codex : 76/20.10 \\
azhardentj@ azhar.edu.eg \\
http://adjg.journals.ekb.eg \\
\hline DOI: $10.21608 /$ adjg.2020.7760.1109 \\
\hline $\begin{array}{l}\text { Pediatric Dentistry \& Orthodontics } \\
\text { (Pediatric Dentistry, Orthodontics) }\end{array}$ \\
\hline
\end{tabular}

\section{KEYWORDS}

$P R P$, canine retraction, root surface, Split mouth.

\begin{abstract}
Purpose: The aim of this study was to assess root surface after platelet rich plasma (PRP) injection as a means for tooth movement acceleration. Materials and Methods: 10 female patients with age ranged between (14-19) years old were selected for this study. Total of 40 canine sites were divided into two groups; each group included 20 canines: (group I): 10 upper canines\& 10 lower canines (right side - study side), (group II): 10 upper canine \& 10 lower canines (left side - control side). All patients were examined to fulfill the inclusion criteria. Patients in each group underwent radiographic evaluation for the root surface after canine retraction (with \& without PRP injection). The primary radiographic variables were the canine retraction rate and the root length after retraction. Results: Study duration for patients in both groups was six months. The difference was statistically significant between the two groups regarding the treatment durations, where it was $3.25 \pm 0.45$ and $5.63 \pm 0.73$ months in PRP group and conventional orthodontic therapies respectively. Actually, no significant changes occurred in clinical probing depth between both groups at the start of treatment and till the end of the $6^{\text {th }}$ month. Group I resulted in decrease in root length by $0.09 \mathrm{~mm} \pm 0.4$, while Group II showed decrease in root length by $1.4 \mathrm{~mm} \pm 0.8$ which is not statistically significant difference. Conclusion: PRP injection is safe easy method of retraction acceleration of the canine without affecting root length.
\end{abstract}

- Paper extracted from Master thesis titled "Evaluation of root surface accompanying platelet rich plasma injection as a technique for orthodontic tooth movement acceleration".

1. BDS 2011, Faculty of Oral and Dental Medicine. Future University(FUE)., Cairo, Egypt

2. Professor of Orthodontics, Orthodontic department-Faculty of Dental Medicine for Girls-Al-Azhar University, Cairo, Egypt

3. Associate Professor of Oral Medicine, Periodontology, Oral Diagnosis and Radiology. Oral Medicine, Periodontology, Oral Diagnosis and Radiology department -faculty of Dental Medicine for Girls Al-Azhar University, Cairo, Egypt

* Corresponding author email: happy_dentist@hotmail.com 


\section{INTRODUCTION}

Orthodontic treatment is usually tooth movement and alignment. This movement have different types as bodily, tipping, intrusion, extrusion, torqueing, up-righting and rotation movements. A study ${ }^{(1)}$ re-examined the present knowledge of the histological changes that occurred with orthodontic tooth movement which was originated in 1904. As he examined histological changes following orthodontics tooth movement in $\operatorname{dogs} \&$ found out that on the pressure side with light force, frontal bone resorption occurred as the osteoclasts resorbed the alveolar bone surface while heavy forces lead to compression of periodontal ligaments leading to hyalinization, formation of cell-free areas. In the hyalinized tissue, undermining bone resorption occurred originated from osteoclasts from adjacent bone marrow areas ${ }^{(2)}$.

This movement depends on remodeling of the bone in osteoblast and osteoclast harmonic reaction which is fully understood ${ }^{(3)}$. Moreover, it was observed that trauma is associated with applied orthodontic forces, even if it was light forces, accordingly light forces are preferable, as they cause minimal trauma to the PDL ${ }^{(4)}$.

Another theory "Biological Electricity Theory" describing orthodontic tooth movement by Piezoelectricity ${ }^{(5)}$ which is defined as " The flow of electric current accompanied the deformation of the crystal structure which seen only in crystalline materials".

A study ${ }^{(6)}$ was done on rats ended with the result of less tooth movement with the massive force application. This result was during tipping movement and also bodily tooth movement. On the other hand, root resorption was more when the force application is more. The resulted increase in root resorption was related to both types of movement, tipping movement and bodily movement of the upper first molar.

The cell - cell interaction often causes root resorption with different amount depends on the case type of treatment, time of the treatment, and the age of the patient ${ }^{(7,8)}$. There are also some other risk factors such as fixed appliances and rectangular wires, also class II elastics could consider as one of these factors that would produce resorption of the root apexes in canines.

Another risk factors as orthognathic surgery, root malformation or short roots, thin and conical shaped roots, or even blunt root apices and finally the root filled teeth ${ }^{(8)}$. A study on 30 adolescents who had orthodontic treatment and they had a root canal treated tooth. All patients were with permanent teeth and having also a class I molar relation with a moderate crowding. The root canal posterior treated teeth didn't finish the treatment with increase Apical Root Resorption after the treatment orthodontically ${ }^{(9)}$. Which gives the results of that the root canal filled teeth could be treated without apical resorption and wouldn't consider as a root resorption risk factor.

Currently, fixed orthodontic treatment requires a long duration for showing a result which poses high risks of caries, external root resorption, and decreased patient cooperation. So, acceleration of the treatment regarding orthodontic tooth movement and shortening of the time is very useful. Now a days, many ways for acceleration of the tooth movement orthodontically have been reported. Such as laser therapy (low-level) or pulsed electromagnetic fields, also as electrical current trails and surgical trails, corticotomy is one of the most popular methods surgically, or even distraction osteogenesis, also the mechanical vibration had been reported with a significant result regarding acceleration techniques.

Among them all, the low laser therapy and corticotomy was the popular, effective and safe methods. Regarding the reported results corticotomy was the most effective of them all and no revel wither other methods as pulsed electromagnetic filed is effective or not as some results was significant while the others wasn't, but on the other hand the dentoalveolar distraction (periodontal distraction) was promising 
regarding acceleration. It needs more evidence regarding the application for acceleration ${ }^{(10)}$.

A new study was to assess when wires or even bands used for anchorage during the orthodontic treatment in the expansion stage for maxillary arch (rapid maxillary expansion) RME. In the study all the first premolars in the maxilla that were subjected to the expansion shower external root resorption ERR. Also, there was partial cementum resorption in some premolars. The study resulted with that the banded and non-banded premolars (anchorage teeth) were giving the same results among the root resorption with no significance reference ${ }^{(11)}$.

Cone Beam Computer Tomography (CBCT) is a reliable diagnostic tool to detect simulated External Apical Root Resorption (EARR), whereas periapical radiography underestimates it. However, if a periapical radiograph is already available to the diagnosis of External Apical Root Resorption (EARR), CBCT should be used with extreme caution to avoid additional radiation exposure ${ }^{(12)}$.

Now a day there is a new way of treatment called the Platelets Rich Plasma (PRP) which is invented in the 1970s and first used in Italy 17 years later in a medical major surgery (open heart) procedure. After that the PRP therapy starts to gain popularity in the 1990s ${ }^{(13,14)}$. Platelet Rich Plasma (PRP) is an autologous source of platelet-derived growth factor and transforming growth factor beta that is formed by platelets concentration by centrifugation. There is many techniques among the centrifugation, by single double or even triple spinning protocols at PRP preparation methods.

This technique produced a concentration of human platelets of $338 \%$ and identified platelet-derived growth factor and transforming growth factor beta within them ${ }^{(15,16)}$.

PRP therapy is exactly the treatment needed to speed healing and reduce time ${ }^{(17)}$. Also, injection of high concentration PRP could accelerate orthodontic tooth movement via decreasing alveolar bone volume density on paradental tissues in a transient way ${ }^{(18)}$. Many studies are done on acceleration of tooth movement by platelet rich plasma (PRP) injection. This can be done by carefully injection of PRP into the canine area. A mixture of PRP and local anesthetic can be injected ${ }^{(19)}$ directly into the periodontal ligament. Afterwards, putting on the brackets and then do the regular treatment plan. This study was to assess root surface after platelet rich plasma (PRP) injection as a means for tooth movement acceleration.

\section{MATERIAL AND METHODS}

The present study was conducted on 12 female patients ages 14-18 years old. These patients selected from those attending the outpatient clinic, Department of Orthodontics, Faculty of Oral and Dental Medicine for Girls, Al Azhar University. All procedures were explained for all patients, informed consent was assigned, and Research and Ethical Committee approval obtained.

Criteria of selection of the participants was fulfilled and subjects were divided into 2 groups:

Group 1: (Test group): Composed of 12 female patients (right side), each canine site in the injected side was considered as a separate entity, with total number of 2 sites requiring periodontal \& bone evaluation as well as orthodontic retraction.

According to planed treatment, they were divided into 2 sub-groups:

Sub group (1 a): Includes 1 site, and in this subgroup the orthodontic treatment was performed in the upper arch of the patient. (UR3)

Sub-group (1 b): Includes 1 site, and in this subgroup the orthodontic treatment was performed in the lower arch of the patient. (LR3)

\section{Group 2: (Control group):}

Composed of 12 female patients (left side of the SAME patients), each canine site in the normally treated side was considered as a separate entity, with 
total number of 2 sites requiring both periodontal evaluation as well as orthodontic retraction.

The aim of including this group was to determine the efficacy of PRP therapy in improving the attachment level, bone fill, and root length as well as bone density in the absence of PRP during orthodontic tooth movement.

According to planned treatment, they were divided into 2 sub-groups:

Sub group (2 a): Includes 1 site, and in this subgroup the orthodontic treatment was performed in the upper arch of the patient. (UL3)

Sub-group (2 b): Includes 1 site, and in this subgroup the orthodontic treatment was performed in the lower arch of the patient. (LL3)

For matching between the study cases and the control, the left side of maxillary and mandibular arches was considered as control, while the right side of both maxilla and mandible was chosen to receive the PRP injection (Injected Side).

\section{Patient Preparation:}

All subjects included in this study were subjected to the following records:

Extra-oral photograph, Intra-oral photograph, Impression of upper and lower arches to prepare orthodontic study, Panoramic radiograph before and after, Lateral cephalometric radiograph before treatment.

Brackets and molar tube bonding of the upper and lower teeth was done using Roth prescription bracket, excepting the premolars intended for extraction.

Levelling and alignment using sequence of (wire 0.014-inch NiTi), (wire 0.016-inch NiTi), (wire 0.016 x 0.022-inch NiTi) ending with (wire 0.018 x 0.022-inch St.St). The last wire remained for 4 weeks before canine retraction. During the levelling and alignment procedure after insertion of 0.16 " $\mathrm{X}$ 0.22 " NiTi, local anesthesia was administrated bilaterally at the buccal sulcus apically between the second premolar and the first maxillary molar, miniscrews were placed in the region between upper second premolar and upper first molar at the junction between the attached gingiva and non- keratinized gingiva. The mini-screws were placed in the region between lower second premolar and lower first molar.

After levelling and alignment, patients were referred for upper and lower first premolar extraction.

Then NiTi Coil spring “ delivering about 150 gm per side was placed on right and left canines after injection of the Platelet rich plasma (PRP) in the both experimental \& control side.

\section{PRP preparation and injection}

Injection and preparation were done in Faculty of Dentistry in Al Azhar University (Girls Branch).

\section{A. Blood Extraction:}

By disposable 22 gouge needle, $5 \mathrm{ml}$ of the venous blood was aspirated from the patient arm veins by trained nurse. The blood drawn directly into two sterile tubes containing anticoagulant (sodium citrate) by the needle.

\section{B. Separation and Concentration of PRP :}

The PRP prepared by the double spin technique as described by previous studies ${ }^{(20,21)}$.

Separation Spin: (first spin) By standard 6 tube holes square bench-top centrifuge the extracted blood centrifuged for 20 minutes at $1.200 \mathrm{rpm}$. This resulted in an opaque red lower fraction, the blood cell component (BCC), and a second upper straw yellow turbid fraction with plasma and platelets, called the serum component (SEC) and in between a buffy layer.

Concentration spin: (second spin) In order to maximize the platelet concentration. a point 
was marked with a marker below the dividing line between these two phases (BCC and SEC), within the BCC. The entire SEC and BCC up to this point were pipetted out into a fresh, sterile tube without anticoagulant. The remaining substance, approximately $0.5 \mathrm{~mL}$ in quantity, was mixed and represented platelet rich plasma (PRP). The PRP separated and aspired into an insulin syringe for injection.

\section{C.PRP injection}

Right side of the both arches was anesthetized, then received 20 units $(0.2 \mathrm{ml})$ of PRP injected intraligamentally in the mesio-buccal, mesio-palatal, disto-buccal and disto-palatal area of the surface of the canines. (5 units each area).

- The experimental side was injected every 4 weeks (28 days). The control side was free of injection.

- Follow up was done every 4 weeks for PRP injections. Also, from the beginning of study, a follow up visit was done every 2 weeks till the end of the 6th month.

\section{Measurements of Teeth Investing Structure:}

An indirect digital image radiographic system; the DBS-Win software, which is a part of vista scan system (Durr Dental Bietigheim), was used to measure the digital panoramic radiographs of the patient before and after the PRP injection, to evaluate the root resorption and, bone density.

\section{Measurement of Root resorption (linear analysis)}

The measurement of the root length was done by measuring the distance between the cementoenamel junction (as a reference point) to the apex of the root (fig 1). A comparison between the linear measurements prior to treatment and 6 months after beginning the orthodontic treatment were done to determine the extent of apical root resorption. The resorption was evaluated in term of millimeters and percentage.

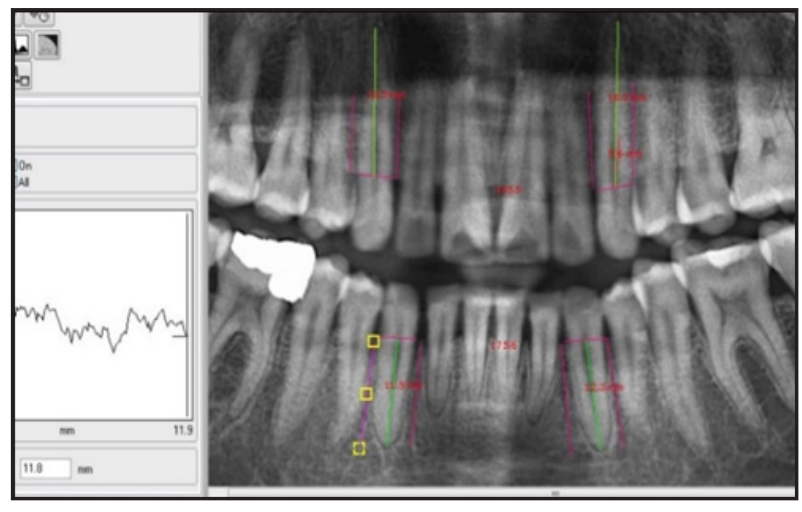

Figure (1) The panoramic x-ray used to measure the bone density \& root length before treatment.

\section{OUTCOMES}

\section{The rate of canine retraction:}

Measured using digital caliper by measuring space from the distal surface of the canine bracket to the mesial surface of the second premolar and measured from plaster models which were poured from alginate impressions of the upper and lower arches. The impressions were taken immediately after extraction of the first premolars and every 2 months till the 4 th month. (3 study models). The rate of canine retraction was measured on both digital caliber and models using $\underline{\mathrm{mm}}$.

\section{Root resorption:}

Root of the upper \& lower canines was measured using after retraction digital panoramic x-ray images \& compared to the pre-retraction images.

\section{RESULTS}

The canine retraction stage took 4 months from the total study time. The difference was statistically significant between the two groups regarding the treatment durations, where it was $3.5 \pm 0.6$ and 5.43 \pm 0.93 months in PRP group and conventional orthodontic therapy respectively in the upper arch \& $3.1 \pm 0.5$ and $5.75 \pm 1$ months in PRP group and conventional therapy respectively in the lower arch. (Table1,2 Fig.2) 
Table (1) Canine retraction ( $\mathrm{mm}$ ) in PRP vs. Control Group in the upper arch and lower arch.

\begin{tabular}{|c|c|c|c|c|}
\hline \multirow{2}{*}{} & \multicolumn{2}{|c|}{ PRP side } & \multicolumn{2}{c|}{ Control side } \\
\cline { 2 - 5 } & Maxilla & Mandible & Maxilla & Mandible \\
\hline Mean & 6.05 & 5.33 & 5.90 & 5.47 \\
\hline SD & 0.57 & 0.73 & 0.58 & 0.73 \\
\hline T & \multicolumn{2}{|c|}{2.69} & \multicolumn{2}{|c|}{1.6} \\
\hline P & \multicolumn{2}{|c|}{$0.013 *$} & \multicolumn{2}{|c|}{$0.124 \mathrm{~ns}$} \\
\hline
\end{tabular}

Table (2) Duration of canine retraction in PRP vs. Control Group in the upper arch and lower arch

\begin{tabular}{|c|c|c|c|c|}
\hline \multirow{2}{*}{} & \multicolumn{2}{|c|}{ PRP side } & \multicolumn{2}{c|}{ Control side } \\
\cline { 2 - 5 } & Maxilla & Mandible & Maxilla & Mandible \\
\hline Mean & 3.5 & 3.08 & 5.42 & 5.75 \\
\hline SD & 0.52 & 0.67 & 0.79 & 0.97 \\
\hline T & \multicolumn{2}{|c|}{1.72} & \multicolumn{2}{|c|}{0.91} \\
\hline P & \multicolumn{2}{|c|}{$0.1003 \mathrm{~ns}$} & \multicolumn{2}{|c|}{$0.37 \mathrm{~ns}$} \\
\hline
\end{tabular}

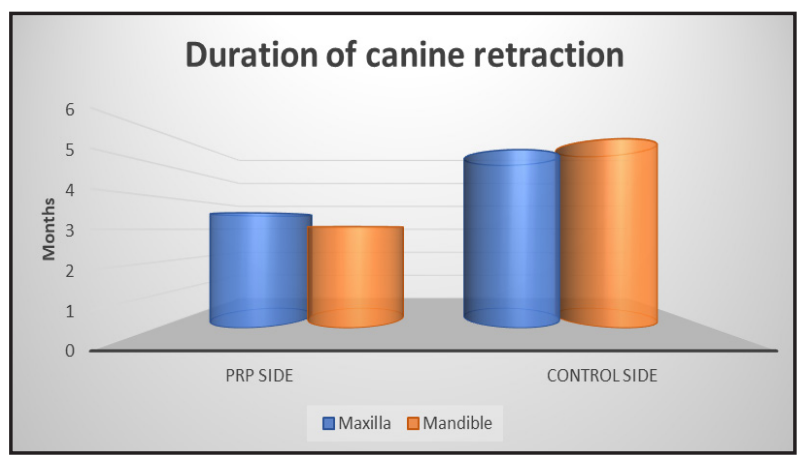

Figure (2) Column chart showing duration of canine retraction in both jaws in PRP and Control side
No significant changes occurred in clinical probing depth between both groups at the start of treatment and till the end of the $6^{\text {th }}$ month.

Group I resulted in decrease in root length by $0.09 \mathrm{~mm} \pm 0.4$, while Group II showed decrease in root length by $1.4 \mathrm{~mm} \pm 0.8$ in the upper arch \& Group I resulted in decrease in root length by $0.09 \mathrm{~mm} \pm 0.4$, while Group II showed decrease in root length by $1.4 \mathrm{~mm} \pm 0.8$ in the lower arch which is not statistically significant difference. (Table 3, Fig. 3)

No significant changes occurred in bleeding index between both groups at the start of treatment and till the end of the $6^{\text {th }}$ month.

When comparing PRP side and conventional retraction side effect on root resorption the effect of both retraction methods on the root length was nearly the same. There was no statically significance difference between both sides concerning this issue.

Upon comparing the maxillary root length changes with the mandibular one in both the PRP injected group and in the conventional one, it showed no statistically significant difference between the degrees of root resorption. The same finding was found in the conventional group, and the clinical results before and after treatment for both arches were significant. (fig.4,5)

Table (3) Root length in PRP vs. Control Group (comparison of both arches)

\begin{tabular}{|c|c|c|c|c|c|c|c|c|}
\hline \multirow{2}{*}{} & \multicolumn{4}{|c|}{ Pre } & \multicolumn{4}{c|}{ Post } \\
\cline { 2 - 10 } & \multicolumn{2}{|c|}{ PRP } & \multicolumn{2}{c|}{ Control } & \multicolumn{2}{c|}{ PRP } & \multicolumn{2}{c|}{ Control } \\
\cline { 2 - 9 } & Upper & Lower & Upper & Lower & Upper & Lower & Upper & Lower \\
\hline Mean & 17.03 & 14.91 & 16.51 & 14.06 & 16.43 & 14.05 & 15.17 & 13.29 \\
\hline SD & 1.96 & 2.31 & 2.15 & 1.63 & 2.09 & 2.26 & 2.26 & 1.75 \\
\hline T & \multicolumn{2}{|c|}{2.42} & \multicolumn{2}{|c|}{3.15} & \multicolumn{2}{c|}{2.68} & \multicolumn{2}{c|}{0.033} \\
\hline P & \multicolumn{2}{|c|}{0.024} & \multicolumn{2}{|c|}{0.0047} & \multicolumn{2}{c|}{0.0137} & \multicolumn{2}{c}{} \\
\hline
\end{tabular}




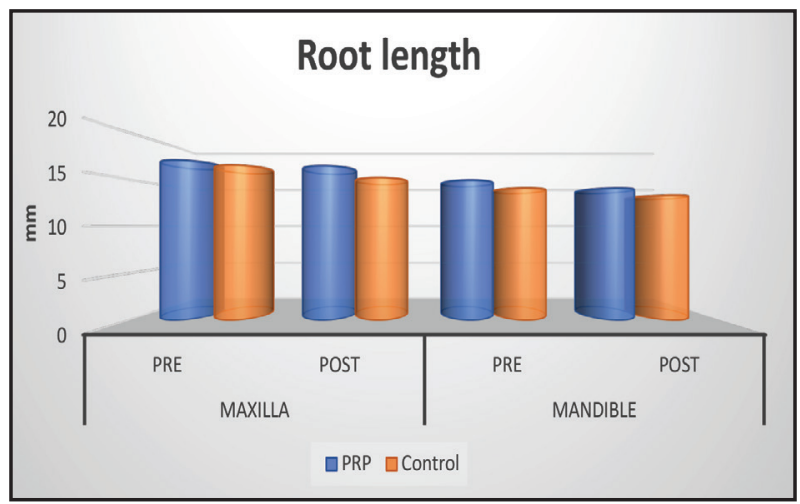

Figure (3) Column chart showing mean value of root length in the upper and lower jaws

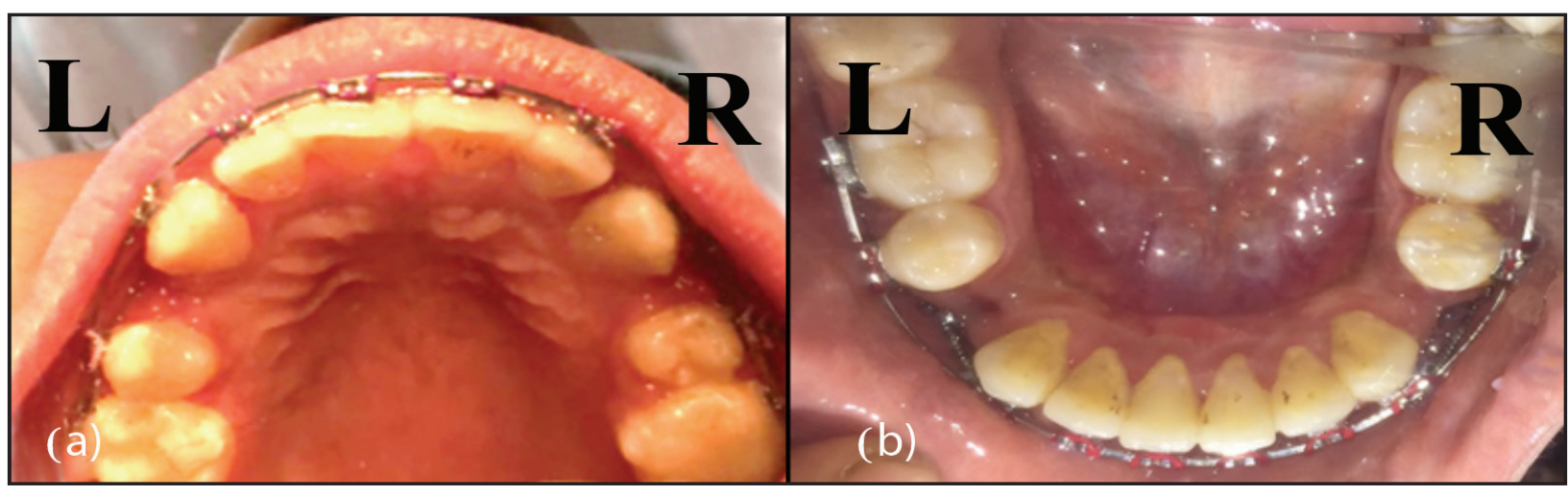

Figure (4) Occlusal view after alignment at the beginning of canine retraction

a. Shows the upper arch (right side: PRP, left side: control)

b. Shows the lower arch (right side: PRP, left side: control)

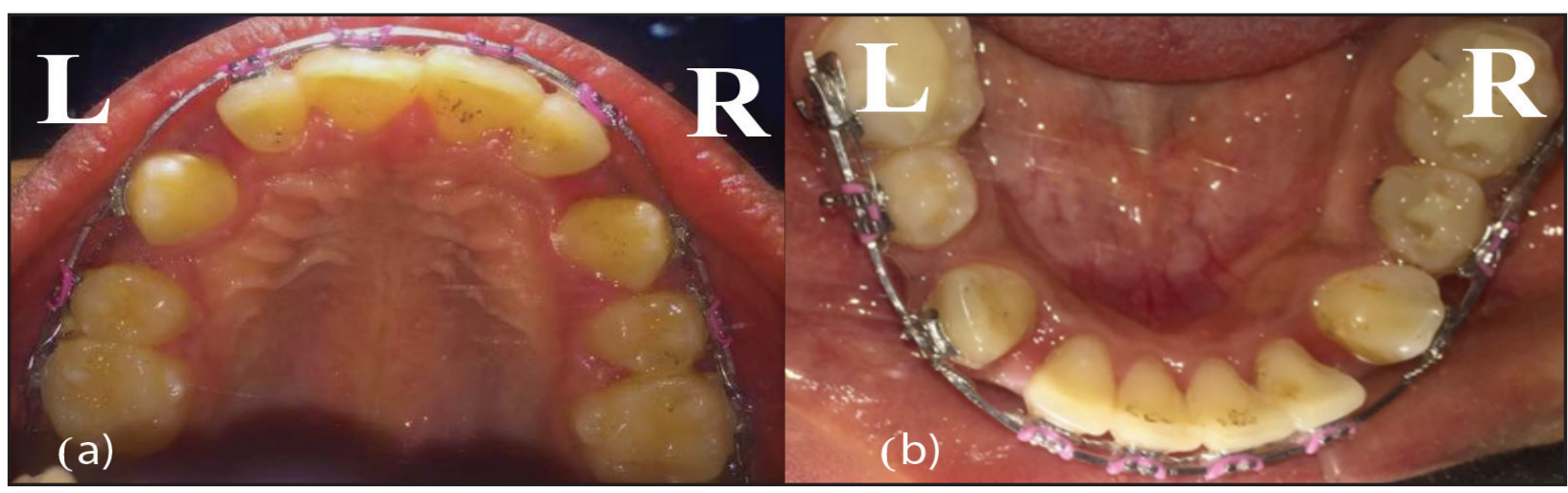

Figure (5) Occlusal view at the end of the study

a. Shows the upper arch (right side: PRP, left side: control)

b. Shows the lower arch (right side: PRP, left side: control) 


\section{DISCUSSION}

Platelet rich plasma (PRP) injection was considered a new trend in OTM acceleration nowadays as it's autogenous nature and healing advantages ${ }^{(19,22)}$.

The injection of PRP was intra-ligamental. The intervals of injection were done every 21 days (three weeks) along the retraction period as recommended by recent studies. To gain the maximum and absolute anchorage, mini-screw placed between second premolar and first molar as mentioned by previous studies with direct ligation to the close coil spring (23-25)

One of the primary out comes of the study was the rate of canine retraction which measured by digital caliper as in previous studies ${ }^{(22,24,25)}$. So, to overcome and minimize the reading errors, three readings were taken, and the mean reading was the final value ${ }^{(24)}$.

The resulted rate of canine retraction in the Maxillary PRP injection side was 1.55 times the control side. This was very similar to the results of the previous studies on the same arch, as the technique was like this study ${ }^{(24-28)}$. Also, it was more than the rate recorded during corticotomy studies, but with the advantage of less invasive technique (28,29).

According to the mandibular arch PRP injected side showed 1.3 times than the control side, which is more than the results of the corticotomy assessted retraction in both maxilla and mandible. The difference in the rate of retraction between the maxilla and the mandible was significant statically [ $\mathrm{p}=$ 0.01], a result of difference in bone nature of the both arches as the mandibular bone is more compact than the maxillary cancellous one as showed in corticotomy study ${ }^{(30)} .1 .4$ to 1.7 -time acceleration was calculated which goes also with the results of this study ${ }^{(29)}$.

Comparing between the maxilla and the mandible the canine retraction in the PRP side was higher in the maxilla, with a significant difference $(\mathrm{p}=0.013)$, and comparing the canine retraction in the control side, a higher result also recorded in the maxilla, with no significant difference $(\mathrm{p}=0.124)$.

Moreover, this PRP injection technique has several advantages, including faster tooth movement, shorter treatment time, safer application and less invasive technique.

Probing depth was assessed using Williams graduated probe as regularly used in assessment of the periodontal condition ${ }^{(33)}$. Result showed a statically significant difference in both sub groups regarding maxilla, as it was $(\mathrm{p}=0.009)$ in the PRP injection side and $(\mathrm{p}=0.025)$ in the control. In the lower arch there was no statically significance regarding pre and post treatment in both sides PRP and control ${ }^{(21)}$. Among both arches same result recorded showing no statically significance.

In the present study root resorption was compared using digital panoramic X-ray and Vista Scene digital programme DWNIS to read and measure changes in root length as previous study ${ }^{(33)}$.

Regarding PRP side and conventional retraction side effect on root resorption the effect of both retraction methods on the root length was nearly the same. There was no difference concerning this issue and it could be due to the short time of the study.

Upon comparing the maxillary root length changes with the mandibular one in both the PRP injected group and in the conventional one, it also showed no difference in root resorption but, statically difference was found in the rate of retraction due to the difference in bone density of the both arches.

\section{CONCLUSION}

From the results of the present study, the following conclusions could be drawn:

PRP injection process is minimally invasive technique that affects tooth movement acceleration positively with more significant effect in maxilla 
than the mandible. PRP injection should be repeated during treatment, as its effect decrease by time. Also, PRP didn't affect the root length significantly during study time in both arches, and finally, the maxillary bone enhancement and tooth movement acceleration by PRP was more significant than the mandible as the density difference of both arches.

\section{REFERENCES}

1. Meilek M.C. The tissue, cellular and molecular regulation of orthodontic tooth movement: 100 years after Carl Sandstedt. Europ J of Orthod. 2006. 28; 221-240.

2. Ariffin Z, Yamamoto Z, Zainol-Abidin IZ, Abdul-Wahab RM. Cellular and molecular changes in orthodontic tooth movement. Sci World J. 2011. 11; 1788-803.

3. D. Roberts - Harry and J. Sandy. Orthodontics. Part 11: Orthodontic tooth movement. Br J Orthod. 2004. 196; 391-4.

4. Storey E. The nature of tooth movement. AJOO. 1973.63; 292-314.

5. Proffit WR, Fields HW, editors. Contemporary Orthodontics. Fifth ed. Mosby Inc: 2013; 250-1.

6. Takako N, Hitoshi H, Megumi H, Irin S, Kotaro A, Takeshi $\mathrm{K}$, et al. Effects of different types of tooth movement and force magnitudes on the amount of tooth movement and root resorption in rats. Angle Orthod: 2014. 84; 1079-85.

7. Hill P A. Bone remodelling. Br J Orthod. 1998; 25: 101-7.

8. Sandy J R, Farndale R W, Meikle M C. Recent advances in understanding mechanically-induced bone remodelling and their relevance to orthodontic theory and practice. Am J Orthod Dento-fac Orthop. 1993; 103: 212-22.

9. Iury C, José V-N, Carlos E. Contribution of cone beam computed tomography to the detection of apical root resorption after orthodontic treatment in root-filled and vital teeth. Angle Orthod. 2015; 85: 771-76.

10. Hu L, Ujjwal P, Yan W, Lina L, Yang Z, and Wenli L. Interventions for accelerating orthodontic tooth movement. Angle Orthod. 2013; 83: 164-71.

11. Débora C. Rapid maxillary expansion: Do banded teeth develop more external root resorption than non-banded anchorage teeth? Angle Orthod. 2016; 86: 39-45.

12. Hongyu R, Jun C, Feng D, Leilei Z, Xiong L, and Yanling D. Comparison of cone-beam computed tomography and periapical radiography for detecting simulated apical root resorption. Angle Orthod. 2013; 83: 189-95.
13. Borrione P, Gianfrancesco AD, Pereira MT, Pigozzi F. "Platelet-rich plasma in muscle healing". Am J Phys Med Rehabil. 2010; 89: 854-61.

14. Petrungaro PS. Using platelet-rich plasma to accelerate soft tissue maturation in esthetic periodontal surgery. Compend Contin Educ Dent 2001; 22:729-36.

15. Marx RE, Carlson ER, Eichstaedt RM, Schimmele SR, Strauss JE, Georgeff KR. Platelet-rich plasma: Growth factor enhancement for bone grafts. Oral Surg Oral Med Oral Pathol Oral Radiol Endod. 1998; 85: 638-46.

16. Frank B. Kelly, MD. Platelet-Rich Plasma (PRP). AAOS. 2013; 21: $739-48$.

17. Marx RE. Platelet-Rich Plasma (PRP): What Is PRP and What Is Not PRP?. Implant Dent.2001. 10; 225-28.

18. Eric J. W. Liou. The development of submucosal injection of platelet rich plasma for accelerating orthodontic tooth movement and preserving pressure side alveolar bone. Asian Pacific Orthodontic Society (APOS) Trends in Orthod. 2016. 6; 5-11.

19. Albanese A., Licata M E., Polizzi B., Campisi G. Platelet -rich plasma (PRP) in dental and oral surgery: from the wound healing to bone regeneration. lmmun Ageing. 2013. 10;21- 23 .

20. Messora M. A standardized research protocol for plateletrich plasma (PRP) preparation in rats. RSBO Rev Sul. 2011; 8:299-304.

21. Khairy Nf., Shendy EE., Askar NA., El-Rouby DH. Effect of platelet rich plasma on bone regeneration in maxillary sinus augmentation (randomized clinical trial). Int J Oral Maxillofac Surg.2013.42:249- 55.

22. Rashid A., EISharabv FA., Nassef EM., Mehanni S., Mostafa YA. Effect of platelet-rich plasma on orthodontic tooth movement in dogs. Orthod Craniofac Res.2017.20;102-10.

23. Nagata MJ., Messora MR., Furlaneto FA., Fucini SE., Bosco AF., Garcia VG.et al. Effectiveness of two methods for prparation of autologous platelet-rich plasma: an experimental study in rabbits. Eur J Dent. 2010.4; 395-402.

24. Said MA. Canine retraction acceleration with platelet rich plasma: A clinical comparative study. A thesis submitted to the Faculty of Dental Medicine, Al-Azhar university (Assiut branch) for partial fulfillment of $\mathrm{PhD}$ in Orthodontics. 2016.

25. Aboul-Ela SM., El-Beialy AR., El-Sayed KF., Selim EN, ElMangoury NH., Mostafa YA. Miniscrew implant-supported 
maxillary canine retraction with and without corticotomy -facilitated orthodontics. Am J Orthod Dentofacial Orthop. 2011.139;252-9.

26. Dixon V., Read MJF., Brien KO., Worthington HV., Mandall NA. A randomized clinical trial to compare three methods of orthodontic space closure. J Orthod. 2002. 29;31-6.

27. Deguchi T., Imai M., Sugawara Y., Ando R., Kushima K., TakanoYamamoto T. Clinical evaluation of a low-friction attachment device during canine retraction. Angle Orthod. 2007.77;968-72.

28. Eltemamy AM. Effect of Platelet Rich Plasma (PRP) Versus Control on the Rate of Tooth Movement in Orthodontic Patients. A thesis submitted to the Faculty of Dentistry, Orthodontic department, Cairo university. 2017.

29. Hayashi K., Uechi J., Murata M., Mizoguchi I. Comparison of maxillary canine retraction with sliding mechanics and a retraction spring: a three- dimensional analysis based on a midpalatal orthodontic implant. Eur J Orthod. 2004. 26; 585-9.
30. Montenegro VC, Jones A, Petocz P, Gonzales C, Darendeliler MA. Physical properties of root cementum: Part 22. Root resorption of the application of light and heavy extrusive orthodontic microcomputed tomography study. Am J Orthod 2012.14;11-9.

31. Appel TR, Potzsch B., Muller J., von Lindern J-J, Berge SJ., Reich RH. Comparison of three different preparations of platelet concentrates for growth factor enrichment. Clin Oral Implants Res. 2002. 13; 22-8.

32. Sammartino G, Tia I, Marenzi G., Espedito Di Lauro A., D'Agostino E., Claudio PP. Use of autologous PlateletRich Plasma (PRP) in periodontal defect treatment after extraction of impacted mandibular third molars. J Oral Maxillofac Surg. 2005. 63; 66-770.

33. Shoman AA. Root surface evaluation after canine retraction by self-ligation brackets. $\mathrm{PhD}$ thesis submitted to Fucalty od dental medicine. Al-Azhar University. 2017. 